\begin{tabular}{|c|c|}
\hline Citation & $\begin{array}{l}\text { Pieter Gijsenbergh, Martina Pepicelli, Christopher L. Wirth, Jan } \\
\text { Vermant, Robert Puers, (2015), } \\
\text { Langmuir monolayer characterization via polymer microtensiometers } \\
\text { Sensors and Actuators A: Physical, 229, 110-117. }\end{array}$ \\
\hline Archived version & $\begin{array}{l}\text { Author manuscript: the content is identical to the content of the } \\
\text { published paper, but without the final typesetting by the publisher }\end{array}$ \\
\hline Published version & http://dx.doi.org/10.1016/j.sna.2015.03.041 \\
\hline Journal homepage & http://www.sciencedirect.com/science/article/pii/S0924424715001648 \\
\hline Author contact & pietergijsenbergh@gmail.com \\
\hline
\end{tabular}

(article begins on next page) 


\title{
Langmuir monolayer characterization via polymer microtensiometers
}

\author{
Pieter Gijsenbergh ${ }^{\mathrm{a}, *}$, Martina Pepicellib,d, Christopher L. Wirth ${ }^{\mathrm{c}, \mathrm{d}}$, Jan \\ Vermant $^{\mathrm{b}, \mathrm{d}}$, and Robert Puers ${ }^{\mathrm{a}}$ \\ ${ }^{a}$ KU Leuven ESAT-MICAS, Leuven, Belgium \\ ${ }^{b}$ ETH Zürich, Department of Materials, Zürich, Switzerland \\ ${ }^{c}$ Cleveland State University, Chemical and Biomedical Engineering Department, \\ Cleveland, Ohio, USA \\ ${ }^{d}$ KU Leuven CIT-SMaRT, Leuven, Belgium
}

\begin{abstract}
A semi-rigid, semi-flexible SU-8 polymer microdevice was designed to measure changes in interfacial tension at an air-water interface. The suspended microtensiometer enclosed a clean air-water interface, with an insoluble surfactant on the exterior. The difference in surface tension between the inside and the outside of the device, called the surface pressure, caused the 850 $\mu \mathrm{m}$ by $3 \mathrm{~mm}$ device to deflect. Finite element simulations were performed to predict device behavior prior to fabrication. Finished devices were tested in a Langmuir trough during multiple compression and expansion cycles using large area changes and slow compression speeds. Shorter experiments subjecting the interface to rapid local monolayer concentration variations were also performed. A platinum Wilhelmy plate was used as an independent surface pressure measurement. The microtensiometer had a theoretical resolution of $0.02 \mathrm{mN} \cdot \mathrm{m}^{-1}$.
\end{abstract}

Keywords: MEMS, SU-8, Tensiometry, Surface pressure, Langmuir monolayers

*Pieter Gijsenbergh - Kasteelpark Arenberg 10, B-3001, Leuven, Belgium - Tel: +32 16325629 - Fax: +32 16321975 - pietergijsenbergh@gmail.com 


\section{Introduction}

The surface tension of a fluid plays a key role in the formation of droplets, insects walking on water, inkjet printing, tears in a wine glass [1] and the Cheerios effect [2]. Moreover, the mechanical and rheological properties of a fluid-fluid interface (of which surface tension is one aspect) are crucial to the production and processing of myriad multiphase materials, which are found in a variety of industrial, engineering and medicinal applications $[3,4,5]$. Often one is interested in the surface tension of an interface populated by a surface-active component as compared to when that interface is clean (i.e. no surfactant). The difference is termed the surface pressure and it increases as the concentration of surface-active component increases (i.e. its mean molecular area decreases). One well-established technique for measuring the surface pressure is to change the surface concentration by compression of the fluid surface and measuring the surface tension with a Wilhelmy plate [6] connected to an electromagnetic balance. For insoluble monolayers, a Langmuir trough $[7,8]$ is often used to compress the interface and to control the surface concentration of surface-active substances [9]. The latter include long-chain surfactants, proteins, fatty acids, phospholipids or even colloidal particles. The setup is somewhat cumbersome to use and requires significant amounts of liquid subphase as well as surface-active components, and may suffer from temperature fluctuations, effects of evaporation, and the large open area makes it prone to contamination. Moreover, the accuracy of the Wilhelmy plate is limited $\left( \pm 0.1 \mathrm{mN} \cdot \mathrm{m}^{-1}\right)$ and its response time is typically longer because of the time constants of the feedback loop of its measurement system.

Besides the Wilhelmy plate, other surface or interfacial tension measurement methodologies are available such as the Du Noüy ring method [10] and the pendant or spinning drop method [11]. Drawbacks of the former technique include the need for a correction factor and the fact that the measurement is performed in a non-equilibrium state of the interface. The latter method is very accurate for low surface tensions $\left(<10^{-2} \mathrm{mN} \cdot \mathrm{m}^{-1}\right)$ but is unsuitable for high values of the interfacial tension.

Miniaturised setups for tensiometry measurements are not entirely new [12] and in this work, we build on earlier ideas by Zell et al. [13] in which a microtensiometer had been proposed, made of a semi-flexible polymer structure. The device described by Zell et al. was a rectangular structure, with thin walls which deflected under the action of surface pressure. The deflec- 
tion of the walls was modeled with the beam equation. In the present work, we develop a device with an embedded read-out system and with the goal to obtain an increased sensitivity. The device described herein is a further step towards routinely measuring the local surface pressure on the microscale, which would greatly benefit the characterization of complex fluid-fluid interfaces. The first section of this article explains the design choices that were made and provides some theoretical background. This is followed by a detailed overview of the microfabrication process for the devices. Before discussing the results in a final section, the experiments and equipment are described.

\section{Theoretical framework and design}

\subsection{Operating principle}

The device consists of two parallel rigid beams, connected by two millimeter-scale springs. Furthermore, there are two shorter beams in the center of the structure to measure the deflection, as will be discussed in section 4.1.2. A $2 \mathrm{D}$ model of the structure in its resting state is presented in figure 1(a). The device can be placed at a fluid-fluid interface so that the inner part of the sensor contains a pristine interface. For all the simulations and experiments discussed in this article, this was a water-air interface. The possibilities of using an oil-water interface are to be explored in future work. Insoluble, surface-active materials can be deposited on the outside of the device, their concentration being varied by dosage or compression, thus creating a surface tension that is different from that of the clean interface. Hence, it results in a surface pressure which causes the device to be compressed through the flexible springs, while the main beams do not noticeably bend. This approach differs from the original design by Zell et al. [13] who relied on the bending of the main beams. The thickness of those beams, combined with the method to measure their deformation, set the sensitivity and the dynamic range of the device. As in the present design the constants of the flexible springs can be altered by both the shape and thickness of the springs, a higher sensitivity can be reached, with the same microfabrication techniques.

The surface pressure $\Pi$ is defined as the change in surface tension between a clean subphase, indicated by $\sigma_{0}$ and that of a surface-active monolayer, denoted simply by $\sigma$. Moreover, the surface tension at the interface after addition of a surface-active component strongly depends on the surface concentration, $\Gamma$. Equation 1 summarizes this definition. 


$$
\Pi(\Gamma)=\sigma_{0}-\sigma(\Gamma)
$$

The subphase in this stage of the tensiometer development was water. Thus, the maximum surface pressure the device should be able to endure is equal to the surface tension of water $\left(\right.$ at $\left.20^{\circ} \mathrm{C}\right)$, i.e. $72.86 \mathrm{mN} \cdot \mathrm{m}^{-1}$ [14]. This constraint determines the minimum required stiffness of the device, as none of the device (spring) edges are allowed to touch each other, even under maximum surface pressure conditions $(\sigma(\Gamma)=0)$. To determine the device compliance, which is dominated by the stiffness of the spring structures, finite element simulations were performed using the COMSOL Multiphysics [15]. Figure 1(b) shows a deformed device under maximum surface pressure conditions.

\subsection{Finite element simulations}

The beam length $l_{\text {beam }}$ and the desired device thickness $t$ were chosen to be $3 \mathrm{~mm}$ and $10 \mu \mathrm{m}$ respectively. These values are comparable to those used in earlier work [13]. The main beam width was fixed at $175 \mu \mathrm{m}$ to ensure the bending of the main beams would be negligible. The stiffness $K$ of the device - for a specific spring geometry - is a function of the Young modulus $E$ of the structural material and its thickness $t$. The Young modulus of SU-8 was assumed to be $2.3 \mathrm{GPa}$ [16], which is slightly higher than the 2 GPa mentioned in the data sheet [17], because the processing conditions (see section 3) were altered [18]. From these design choices, the dimensions of the spring structures were optimized via simulations using the maximum surface pressure constraint described at the end of section 2.1. All the main in-plane dimensions of the microtensiometer are indicated in figure 1(a).

To study the impact of process variations - specifically the device thickness - on the device compliance, simulations were performed for several values of $t$, around the chosen thickness of $10 \mu \mathrm{m}$. The results of these simulations are shown in figure 2. It is clear that as the device thickness, and with that also $K(E, t)$, increases, the deformation for a given surface pressure is lower. Equation 2 describes the linear tensiometer deflection $\Delta y$ as a function of the surface pressure $\Pi$.

$$
K(E, t) \cdot \Delta y=2 \cdot l_{\text {beam }} \cdot \Pi(\Gamma)
$$


This equation solely contains parameters stemming from the device geometry and the material used for the fabrication. For any observed deflection, the surface pressure $\Pi$ can be directly deduced from this equation without the use of any adjustable or fitting parameters, on the condition that the Young modulus and thickness are both known. Results obtained using the microtensiometers can thus be compared to other, independent measurement techniques such as the Wilhelmy plate. For the aforementioned Young modulus of $2.3 \mathrm{GPa}$, spring dimensions as indicated in figure 1(a) and a verified device thickness $t$ (post-fabrication, using a Dektak XT profilometer) of 10.5 $\mu \mathrm{m}$, the simulated stiffness $K(E, t)$ is $2.95 \mathrm{~N} / \mathrm{m}$, i.e. a compliance of 2.03 $\mu \mathrm{m} /\left(\mathrm{mN} \cdot \mathrm{m}^{-1}\right)$.

\section{Fabrication process}

The fabrication of the microtensiometers starts with the thorough cleaning of a 3-inch silicon substrate in Piranha, a 3-to-1 mixture of sulphuric acid and hydrogen peroxide. After rinsing the substrate with deionized (DI) water and dehydrating it on a hot plate, a $2 \mu \mathrm{m}$ layer of LOR10B from Chimie Tech Services is spin coated at $1500 \mathrm{rpm}$ for 60 seconds and subsequently baked for 5 minutes on a hot plate at $170^{\circ} \mathrm{C}$. A layer of S1818 positive photoresist from micro resist technology $\mathrm{GmbH}$ is spin coated on top of the LOR10B at $4000 \mathrm{rpm}$ for 30 seconds and baked for 2 minutes at $115^{\circ} \mathrm{C}$. Thereafter, this layer is selectively exposed to $40 \mathrm{~mJ} / \mathrm{cm}^{2}$ of UV light using a first photomask. The exposed S1818 is developed in 3-to-1 diluted 351-developer from micro resist technology GmbH. The LOR10B is patterned during this step as well. After rinsing and drying the substrate, the remaining S1818 is stripped from the silicon wafer in propylene glycol monomethyl ether acetate (PGMEA), rinsed in isopropyl alcohol (IPA) and DI water, and finally dried. Sacrificial islands of LOR10B remain on the substrate, as illustrated by figure 3(a).

Following a HF dip to ensure a hydrophobic silicon surface, $10 \mu \mathrm{m}$ of SU-8 2010 from micro resist technology $\mathrm{GmbH}$ is spin coated at $3000 \mathrm{rpm}$. A soft bake of 7 minutes at $95^{\circ} \mathrm{C}$ precedes the selective exposure of SU-8 to $125 \mathrm{~mJ} / \mathrm{cm}^{2}$ of UV light. The second photomask is carefully aligned over the sacrificial pattern on the substrate using an EVG®620 mask alignment system. A post-exposure bake is performed at $95^{\circ} \mathrm{C}$ and followed by a 90 second development in PGMEA. A one-hour hard bake at $150{ }^{\circ} \mathrm{C}$ is necessary to complete the curing process. The final SU-8 structures consist of a tensiometer, a triangular suspension and a square anchor on the substrate, as 
shown in figure $3(\mathrm{~b})$. The sacrificial layer is removed using a copious amount of 3-to-1 diluted 351-developer, and a critical point drying with $\mathrm{CO}_{2}$ is performed to achieve a stictionless release, as shown in figure 3(c). To detach a tensiometer from the substrate, a needle with a droplet of glue (Permabond 102 Industrial Cyanoacrylate Adhesive) is lowered onto the triangular suspension using a micropositioner. Once the glue is dry, the connection to the square anchor is severed and the tensiometer is lifted off the substrate.

\section{Experimental details}

Figure 4 shows the entire setup during a single tensiometer test. The main parts of the setup are indicated and some explanation is provided in section 4.1. Two different types of experiments were performed and are elucidated in subsections 4.2 and 4.3 .

\subsection{Equipment}

\subsubsection{Wilhelmy plate and Langmuir trough}

Testing was performed in a Langmuir trough that allowed for the modification of the surface concentration of hexadecanol, an insoluble surfactant, via barrier movement. An independent measurement of the surface pressure was performed with a Wilhelmy plate, suspended as close to the tensiometer as the setup and capillary length would allow. The Wilhelmy plate is made of platinum and has a length $l_{p}$ and a width $w_{p}$. It has a total mass of $m_{p}$ and was suspended from a microbalance, through the air-water interface, thereby being submerged for a volume $V_{s}$. The total force registered by the microbalance consists of three components, as summarized in equation 3.

$$
F_{\text {Wilhelmy }}=F_{\text {gravity }}+F_{\text {interface }}-F_{\text {buoyancy }}
$$

The gravitational force is a result of the plate mass, while the buoyancy force stems from the partial submersion of the Wilhelmy plate. The component $F_{\text {interface }}$ in equation 3 originates from the surface tension $\sigma$ at the interface surrounding the entire plate. The expanded equation thus becomes

$$
F_{W i l h e l m y}=m_{p} g+2\left(l_{p}+w_{p}\right) \sigma \cos (\theta)-\rho_{l} V_{s} g
$$

where $\rho_{l}$ is the density of the subphase.

As the mass of the plate is known prior to the experiment and the submerged 
plate volume in the liquid can be calculated, both contributions can be calibrated out. The only remaining unknown in the measured force is then the surface tension $\sigma$. Assuming that the contact angle $\theta$ of the wetting liquid is $0^{\circ}$, equation 4 can be simplified to

$$
\sigma=\frac{F}{2\left(l_{p}+w_{p}\right)}
$$

Considering the definition of surface pressure, equation 1, it follows that after registering the surface tension of the clean subphase ( $\sigma$ in equation 5 is then $\sigma_{0}$ ), the Wilhelmy plate can be used to quantify the surface pressure of an interface containing insoluble material. Equation 5 also implies that the accuracy of this method (generally $0.1 \mathrm{mN} \cdot \mathrm{m}^{-1}$ ) is determined by the accuracy of the force measurement and the size of the Wilhelmy plate.

\subsubsection{Imaging setup and edge-tracking algorithm}

A tensiometer was glued on a needle tip, lowered onto the clean air-water interface and carefully positioned over a small transparant window in the Langmuir trough using a micropositioner, as can be seen in figure 4. Underneath the window was the objective of an inverted microscope, equipped with a camera, to register the deflection of the tensiometer during testing. The magnification of the objective (x20) was too large to contain the entire device, so a device picture from a scanning electron microscope (SEM) is shown in figure 5 to show the read-out mechanism embedded in the tensiometer more clearly. It consists of two short beams at the center of the device. The innermost edges of these beams are the ones in the view of the inverted microscope, as can be seen in figure 6(a). Optical micrographs of this embedded read-out system were recorded at a frequency of 0.5 or $1 \mathrm{~Hz}$ during the experiments. Although the camera was able to capture images at higher frame rates (which would increase the time resolution of the measurement), only a limited amount of images could be stored during a single recording.

The micrographs were first processed in ImageJ [19] by converting grayscale images to binary and subsequently blurring them with a Gaussian filter [20] of standard deviation 5, which was chosen to minimize the error in the mean of the fit. Image filtering is commonly used to achieve sub-pixel accuracy in particle and edge tracking. The output from ImageJ was a vector of pixel intensities along a line perpendicular to the tracked edge. An algorithm 
implemented in MATLAB fit a Gaussian curve to the vector of pixel intensities, thereby identifying the location of the center of each edge for each micrograph. This process is illustrated in figure 6(b). Finally, the deflection between the centers of the two edges was calculated in pixels and converted to microns with the conversion factor of 0.315 microns/pixel. This process was conducted for each micrograph of a series in time, thus producing the edge deflection (and device compression) as a function of time. The error in position of the tracked edge stated herein is based on the standard error in the mean of the Gaussian curve fit. Although this filtering process allowed for an accurate determination of the center of each tracked edge and, ultimately surface pressure, there may be possible improvements. For example, applying a Gaussian filter to the grayscale image (rather than binary) will further improve the accuracy of the tracking algorithm, but this requires better quality images than were obtained with the current camera/lens combination.

\subsection{Linear compression and expansion of Langmuir monolayer}

The Wilhelmy plate was suspended through the clean interface and the balance was zeroed. The tensiometer was lowered onto the clean interface and positioned over the microscope objective. After introducing $20 \mu \mathrm{L}$ of the hexadecanol solution $(1 \mathrm{mg} / \mathrm{mL}$ in isopropanol) in the Langmuir trough, the surface concentrations were allowed to equilibrate for 30 minutes. The experiment was initiated by displacing the trough barriers at a speed of 15 $\mathrm{mm} / \mathrm{min}$, thereby varying the mean molecular area (MMA) of the hexadecanol from 47.5 to $20.4 \AA^{2} /$ molecule, and back. This barrier displacement speed is slow enough for hexadecanol to reach equilibrium. The compressionexpansion cycle was repeated three times and the total duration of these three cycles was 35 minutes. During this time, micrographs of the embedded readout structure were recorded at a frame rate of $0.5 \mathrm{~Hz}$, while the Wilhelmy plate sampled the surface pressure at $1 \mathrm{~Hz}$.

\subsection{Oscillatory compression of Langmuir monolayer}

The setup for this experiment was identical to that described in the first paragraph of section 4.2. The barriers were closed until they were $95 \mathrm{~mm}$ apart. Subsequently, the barriers were oscillated around this position at a frequency of $62.5 \mathrm{mHz}$, with a predescribed amplitude. Initially, an enclosed trough area change of $1 \%$ was used. For a total of 16 oscillations, the entire experiment took just over 4 minutes. Both the Wilhelmy plate and the camera recorded data at a frequency of $1 \mathrm{~Hz}$ during this time. Separated 
by 15-minute intervals, the oscillation experiment was repeated twice with the same microtensiometer, but with increased area changes of $2 \%$ and $3 \%$. All other parameters remained the same. Table 1 summarizes the calculated minimum and maximum MMA for the hexadecanol during these three tests.

\section{Results}

The results of the measurements described in section 4.2 are presented in figure 7. The data collected by the Wilhelmy plate is set out in black (left $y$-axis) as a function of time, while the deflection of the tensiometer (right $\mathrm{y}$-axis) is presented in red. The conversion factor between the two y-axes is the stiffness $K(E, t)$ as predicted by the simulations performed prior to the experiment (i.e. $2.03 \mu \mathrm{m} /\left(\mathrm{mN} \cdot \mathrm{m}^{-1}\right)$, see section 2.2$)$.

The results from both devices show a good correlation as both data sets practically overlap. This is remarkable in the sense that the micrographs were processed using only geometric parameters inherent to the equipment, and the conversion factor obtained from simulations. No additional adjustable or fit parameters were used. The Young modulus $(E=2.3 \mathrm{GPa})$ along with the measured device thickness $(t=10.5 \mu \mathrm{m}$ for these devices) are thus sufficient to predict the device behavior. This is interesting in case other device or spring geometries want to be designed. However, as the experiment progresses, a growing offset appears between the results from both measurement devices. This growing discrepancy is attributed to evaporation of the subphase, hence an error on the side of the microbalance to which the Wilhelmy plate is attached (see equation 3) due to a change of the buoyancy. As subphase liquid evaporates, the buoyancy on the plate decreases and thus the registered force increases. This in turn leads to a lower surface pressure output value. Subphase evaporation causing the liquid level in the Langmuir trough to drop by just $200 \mu \mathrm{m}$ is already sufficient to explain a measurement difference of $1 \mathrm{mN} \cdot \mathrm{m}^{-1}$, as is the case in the results presented in figure 7 .

Figure 8 shows the results of three consecutive oscillation experiments using the same microtensiometer. Again, without having used any adjustable parameters, an excellent correlation between the Wilhelmy plate and tensiometer measurements is clear. Moreover, these experiments show the tensiometer is able to follow the rapidly varying surface pressure. It can be noted here that the material used to fabricate the tensiometer, SU-8, as well as the surrounding liquids cause viscoelastic damping of the tensiometer response. This effect is not noticeable in the results presented in figure 8 , but it may 
emerge if a significantly higher oscillation frequency is used. An in-depth investigation of the response time of these microtensiometers could be the subject of future work, but exploratory simulations predict it to be in the order of tens of milliseconds.

In the final two experiments, shown in figures 8(b) and 8(c), the effect of the evaporation on the Wilhelmy plate once again becomes clear. Even though the experiments themselves are short, the delay between them allows the subphase time to evaporate.

Finally, these graphs also show the tensiometer is able to pick up very small surface pressure changes. This is especially clear in figure 8(a) where the total surface pressure change is only just over $1 \mathrm{mN} \cdot \mathrm{m}^{-1}$. Obviously, the actual accuracy of the microtensiometer depends strongly on the initial quality of the micrographs. Using the conversion factor of the microscope objective $(0.315 \mu \mathrm{m} /$ pixel $)$, the tensiometer sensitivity $\left(2.03 \mu \mathrm{m} /\left(\mathrm{mN} \cdot \mathrm{m}^{-1}\right)\right)$ and the edge tracking accuracy $( \pm 0.1$ pixel based on the standard error in the fit of the Gaussian curve of standard deviation 5), one can calculate the maximum resolution of this technique to be around $0.02 \mathrm{mN} \cdot \mathrm{m}^{-1}$. This is roughly five times more accurate than the classic Wilhelmy plate. Moreover, this is still a fairly conservative estimate for the potential accuracy of this technique. Using a higher magnification objective, decreasing the device stiffness or increasing the main beam lengths for instance are just some of the options that can be explored to increase the sensitivity even further.

\section{Conclusion}

Semi-flexible microtensiometers were designed and tested to measure local surface pressure variations of an insoluble model surfactant. The device encloses a clean air-water interface, while its outer perimeter is exposed to an interface containing an insoluble component. This difference in surface tension between the outside and the inside results in a linear deflection of the device. The device was subjected to two types of experiment: long tests where the surface concentration of insoluble component was gradually varied, and shorter trials where the response to rapid oscillations was observed. In both cases, the data collected from the tensiometer was very strongly correlated with an independent measurement using a Wilhelmy plate. The geometry of the microtensiometers, along with the image processing algorithm that was used, allows a maximum resolution of $0.02 \mathrm{mN} \cdot \mathrm{m}^{-1}$. The device described herein is a further step towards the local measurement of surface pressure 
and surface stress of planar interfaces on the microscale, which would greatly benefit the characterization of complex fluid-fluid interfaces.

\section{Acknowledgements}

This research has been funded by Interuniversity Attraction Poles Programme (IAP 7/38 MicroMAST) initiated by the Belgian Science Policy Of-

fice. Supported by the Hercules initiative for large equipment ("HELICOM", ZW11-15).

\section{References}

[1] C. Marangoni, Sull espansione delle goccie, sulle superfici liquide, II Nuovo Cimento 2 (1870) 105-120.

[2] D. Vella, L. Mahadevan, The "Cheerios effect", Am. J. Phys. 73 (2005) $817-825$.

[3] B. S. Murray, Stabilization of bubbles and foams, Curr. Opin. Colloid Interface Sci. 12 (2007) 232-241.

[4] J. Zasadzinski, J. Ding, H. Warriner, F. Bringezu, F. Waring, The physics and physiology of lung surfactants, Curr. Opin. Colloid Interface Sci. 6 (2001) 506-516.

[5] G. McHale, M. Newton, Liquid marbles: principles and applications, Soft Matter 7 (2011) 5473-5481.

[6] L. Wilhelmy, Ueber die Abhängigkeit der Capillaritäts-Constanten des Alkohols von Substanz und Gestalt des benetzten festen Körpers, Annalen der Physik 119 (1863) 177-217.

[7] A. Pockels, Surface tension, Nature 43 (1891) 437-439.

[8] I. Langmuir, The constitution and fundamental properties of solids and liquids. II. Liquids, Journal of the American Chemical Society 39 (1917) 1848-1906.

[9] J. Drelich, C. Fang, C. White, Measurement of interfacial tension in fluid-fluid systems, Encyclopedia of surface and colloid science (2002) 3152-3166. 
[10] P. L. du Noüy, An interfacial tensiometer for universal use, The Journal of General Physiology 7 (1925) 625-633.

[11] B. Vonnegut, Rotating bubble method for the determination of surface and interfacial tensions, Rev. Sci. Instrum. 13 (1942) 6-9.

[12] Y. Hu, K. Y. C. Lee, J. Israelachvili, Sealed minitrough for microscopy and long-term stability studies of Langmuir monolayers, Langmuir 19 (2003) 100-104.

[13] Z. A. Zell, S. Q. Choi, L. G. Leal, T. M. Squires, Microfabricated deflection tensiometers for insoluble surfactants, Applied physics letters 97 (2010).

[14] N. Pallas, Y. Harrison, An automated drop shape apparatus and the surface tension of pure water, Colloids and Surfaces 43 (1990) 169-194.

[15] COMSOL Multiphysics, http://www.comsol.com/, Online (verified October 21, 2014).

[16] K. Wouters, R. Puers, Determining the Youngs modulus and creep effects in three different photo definable epoxies for MEMS applications, Sensors and Actuators A: Physical 156 (2009) 196-200.

[17] SU-8 2000 Permanent Epoxy Negative Photoresist, http://www. microchem.com/pdf/SU-82000DataSheet2000_5thru2015Ver4.pdf, Online (verified October 21, 2014).

[18] R. Feng, R. J. Farris, Influence of processing conditions on the thermal and mechanical properties of SU8 negative photoresist coatings, J. Micromech. Microeng. 13 (2003) 80-88.

[19] ImageJ, http://imagej .net/, Online (verified October 21, 2014).

[20] J. C. Crocker, D. G. Grier, Methods of digital video microscopy for colloidal studies, Journal of colloid and interface science 179 (1996) 298-310. 


\section{Vitae}

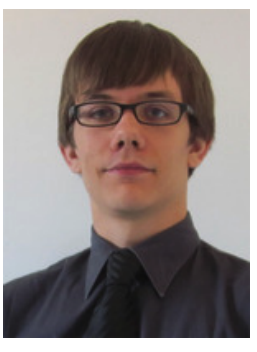

Pieter Gijsenbergh was born in Leuven, Belgium, in 1986. He obtained his degree of Master in Electrical Engineering in 2010 at the KU Leuven with a thesis on "Manufacture and measurement of polymer MEMS for material characterization". Resulting publications were among the first to characterize EpoClad, a polymer similar to SU-8. Since October 2010 he is working as a researcher at the MICAS group of the KU Leuven, under the guidance of Prof. Puers, toward obtaining a PhD in Electrical Engineering. His main research is focused on the microfabrication of devices for microfluidics, including but not limited to capacitance sensors. Until May 2012, he worked on the development of capacitive void fraction sensors for microfluidics. Since then, he has been involved in a project centered around sensing and manipulating surface tension effects and capillary forces.

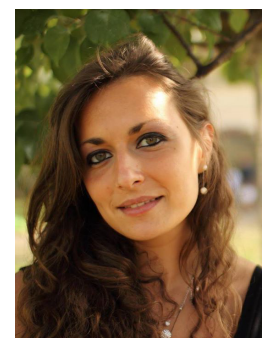

Martina Pepicelli was born in Napels, Italy, in 1988. She obtained her degree of Master in Chemical Engineering in 2013 at the University of Naples Federico II with a thesis in Computational Fluid Dynamics: "Photobioreactors, effects of operating conditions". This thesis work was carried out in collaboration with University College of London, where she spent 5 months. Since September 2013 she is working as PhD researcher under the guidance of Prof. Jan Vermant, for one year at Soft Matter Rheology and Technology Lab (SMaRT) at KU Leuven, Belgium and currently at ETH Zurich. Her research focuses on the structure and rheology of soft matter interfaces.

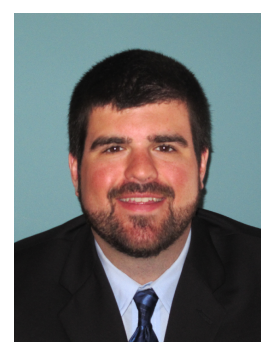

Christopher L. Wirth received his $\mathrm{PhD}$ in Chemical Engineering at Carnegie Mellon University (CMU) in 2012, with work on the directed assembly of colloidal particles in an electric field. He also spent time as a research chemist at PPG Industries in conjunction with the Center for Complex Fluids at CMU. He was the recipient of the Ken Meyer Award for Excellence in Graduate Research, a Bertucci Graduate Fellowship at CMU, and an Achievement Rewards for College Scientists Scholarship. From early 2013, he was a postdoctoral scholar in the Soft Matter, 
Rheology and Technology Lab (SMaRT) at KU Leuven, Belgium, where he worked on the hierarchal assembly of nanoparticles onto chemically modified substrates. He is currently an assistant professor in the Chemical and Biomedical Engineering Department at Cleveland State University in Ohio, where his research topics include colloid and interface science, electrokinetics, and nanotechnology.

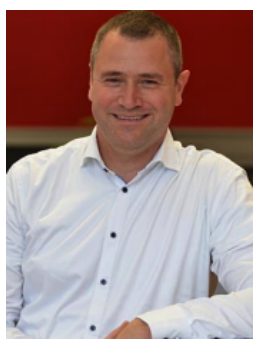

Jan Vermant studied Chemical Engineering at the KU Leuven graduating with a $\mathrm{PhD}$ in 1996. He then spent quite some time abroad, as a visiting scholar, postdoctoral researcher at Stanford University, The university of Delaware and CNRS labs in Sophia Antipolis and Bordeaux. In 2000 he became an Assistant Professor at KU leuven, where he became full professor in 2005. He was a visiting assistant professor at Stanford University in 2001, visiting scholar at Princeton University in 2006 and visiting Michelin professor at the ESPCI (F) in 2011. He moved to ETH in August 2014 but is still associated with KU Leuven. He is an author or co-author of over 140 papers, including book chapters. He was a recipient of the Dupont Young Faculty award (2002) and the FWO Exxon-Mobil European Science and Engineering Award (2007) and is the European editor of Rheologica Acta. He is also a Fellow of the Royal Society of Chemistry. Prof. dr. Vermant currently holds the Professorship in Soft Materials at the ETH Zürich, in the Department of Materials (D-MATL).

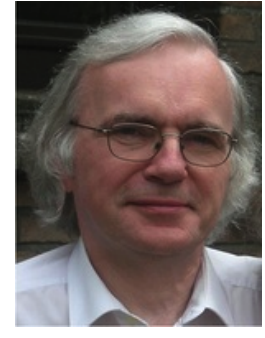

Robert Puers received his PhD in 1986 at the KU Leuven, where he created the clean room facilities for silicon and hybrid circuit technology at ESAT-MICAS in 1984. He is a European pioneer in the research on micromachining, MEMS and packaging techniques, mainly for biomedical implantable systems. More recently, microfluidic, optical and polymer MEMS were added to his research. In addition, his general interest in low power telemetry, with the emphasis on low power intelligent interface circuits and on inductive power and communication links has promoted the research of the ESAT-MICAS laboratory to international recognition. At present, he is a full professor at the KU Leuven, teaching several courses in microsystems, sensors and biomedical instrumentation. The clean room facilities still run under his guidance. He has (co-)authored over 600 papers in reviewed journals or international conferences, was general 
chair of Eurosensors between 2004 and 2010 and acted as General Program Chairman for Transducers 2007. He is also an IEEE and IoP fellow. 

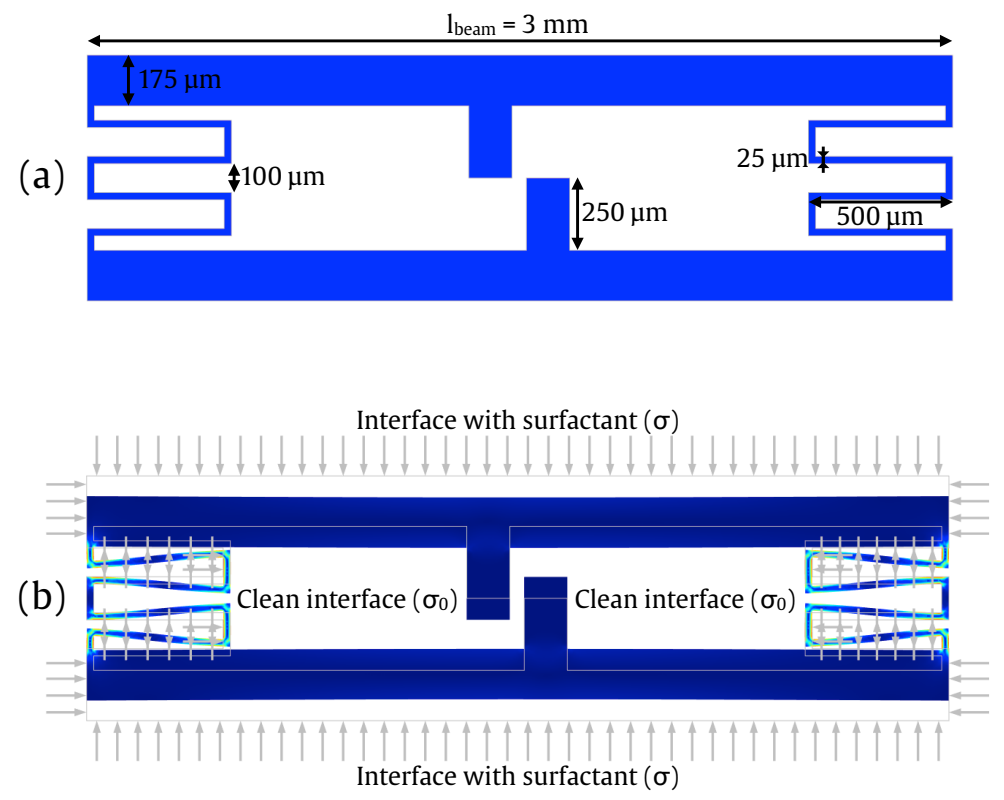

Figure 1: 2D model of the SU-8 tensiometer. (a) The device in its neutral state with an indication of the main dimensions. (b) A surface pressure deforming the structure is simulated as a uniformly distributed external load, perpendicular to all device boundaries.

Table 1: Minimum and maximum mean molecular areas during rapid, local oscillation experiments.

\begin{tabular}{c||c|c} 
Area change & MMA $_{\min }$ & $\mathrm{MMA}_{\max }$ \\
\hline \hline $1 \%$ & $18.46 \AA^{2} /$ molecule & $18.82 \AA^{2} /$ molecule \\
\hline $2 \%$ & $18.24 \AA^{2} /$ molecule & $18.97 \AA^{2} /$ molecule \\
\hline $3 \%$ & $18.01 \AA^{2} /$ molecule & $19.11 \AA^{2} /$ molecule
\end{tabular}




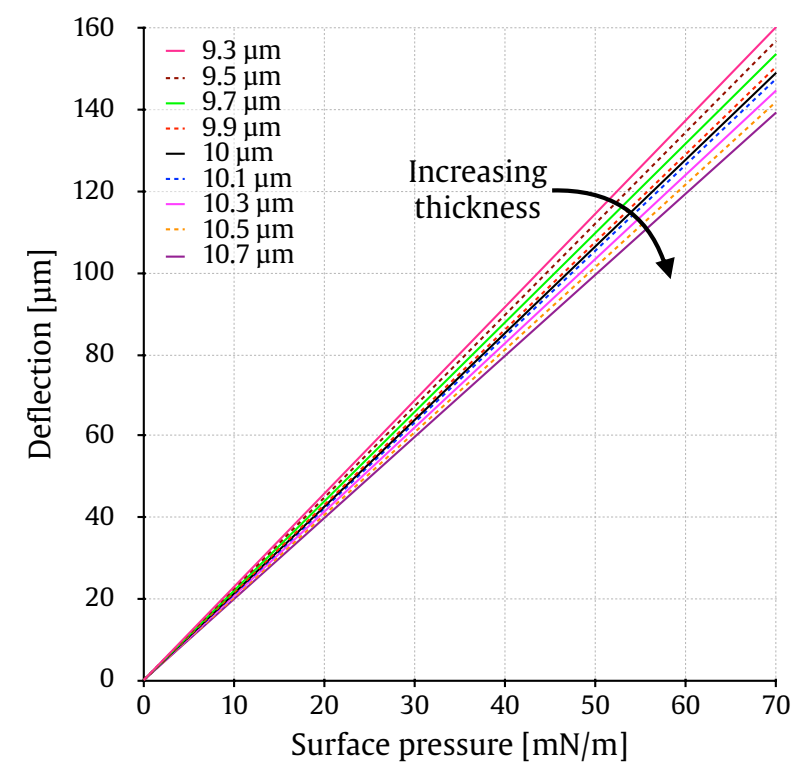

Figure 2: Simulated deflection of an SU-8 tensiometer for various device thicknesses around the anticipated thickness of $10 \mu \mathrm{m}$. 


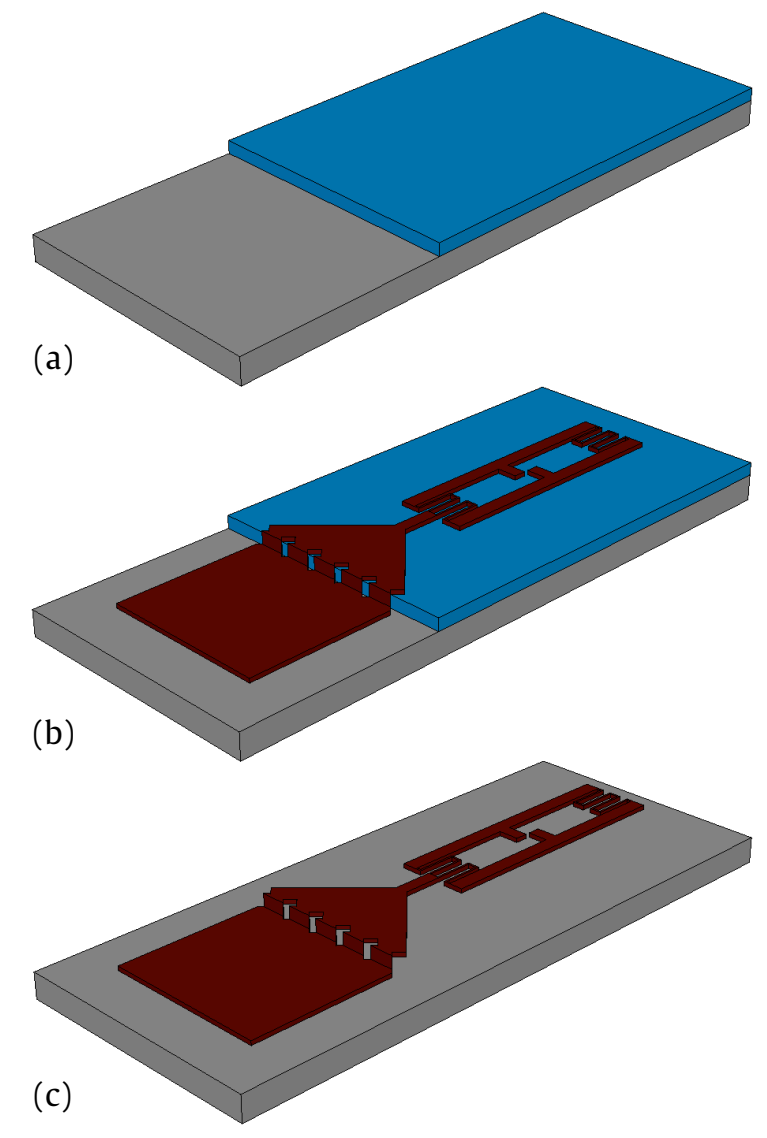

Figure 3: Fabrication process steps. (a) The patterned sacrificial layer. (b) The patterned device layer. (c) The released structure. 


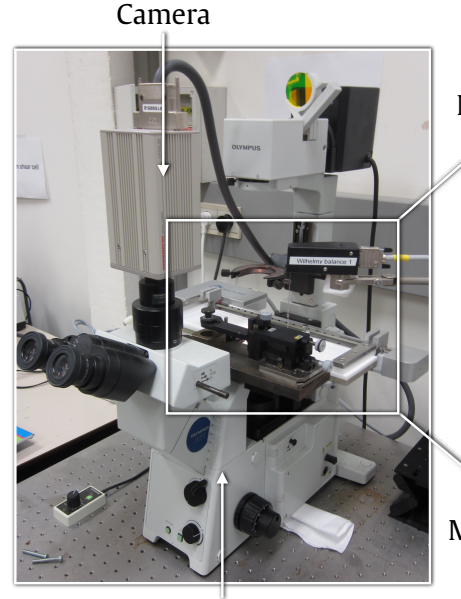

Inverted microscope (x20)

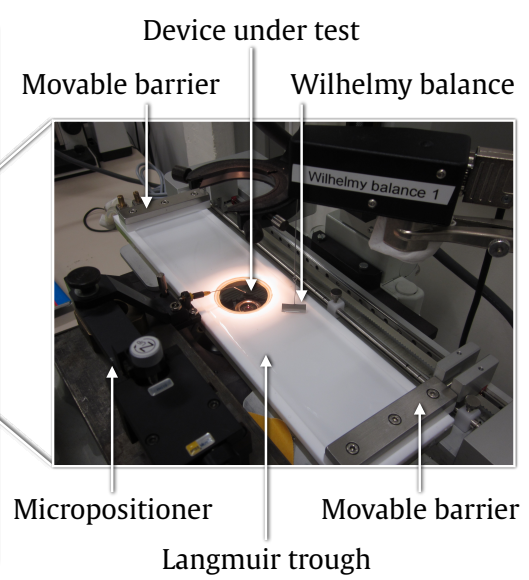

Langmuir trough

Figure 4: Test setup showing Wilhelmy balance, Langmuir trough and inverted microscope setup along with the device under test, held in place above the microscope lens by a needle connected to a micropositioner.

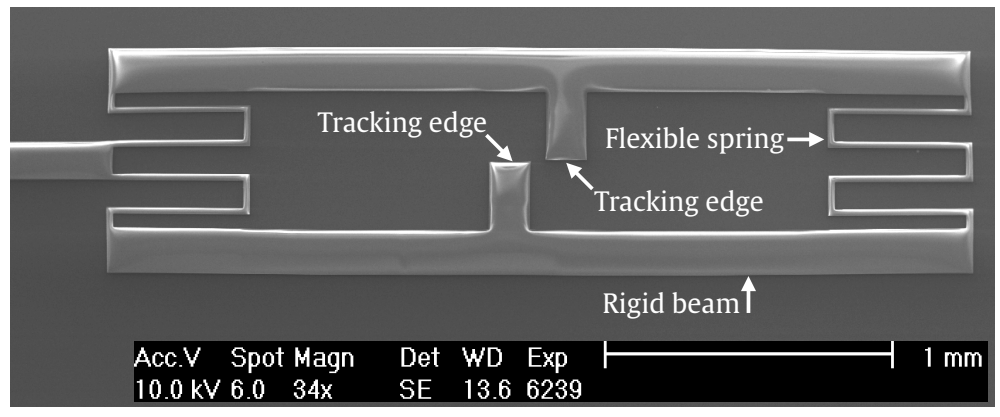

Figure 5: SEM image of an unreleased SU-8 tensiometer with embedded read-out mechanism consisting of two beams. To the left, the suspension beam that connects the tensiometer to the triangular patch where a needle can be glued is visible. 


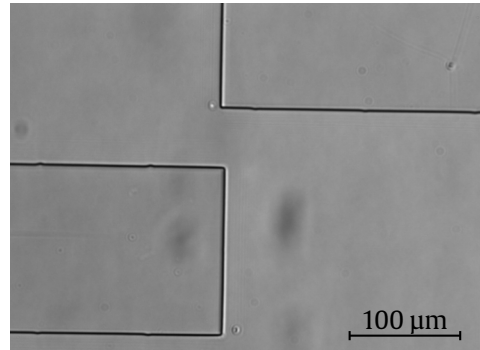

(a)

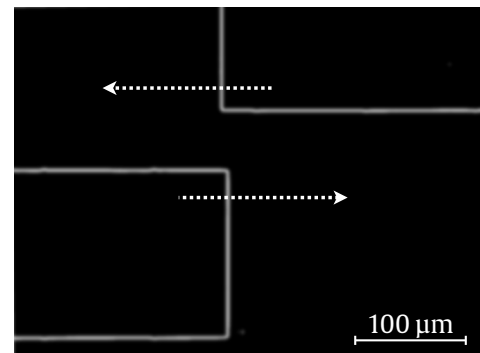

(b)

Figure 6: Read-out mechanism of a microtensiometer. (a) Raw grayscale micrograph as registered by the camera during an experiment. (b) Filtered binary image with arrows indicating where the edge movement is tracked by the algorithm.

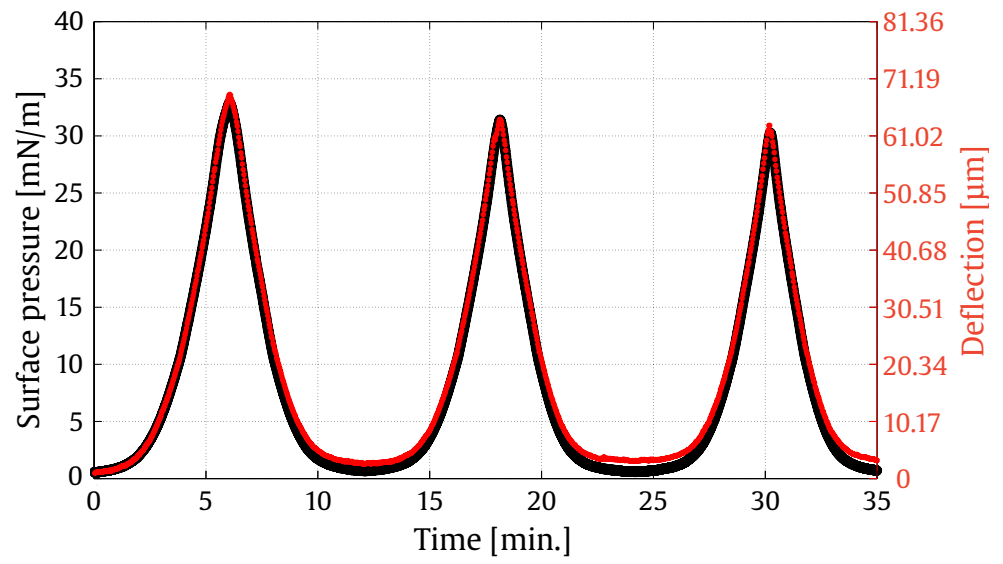

Figure 7: Measurement results showing the surface pressure measured by a Wilhelmy plate (black) and the tensiometer deflection (red) during three compressions/expansions in a Langmuir trough. As the right axis ticks were obtained from the left axis using the simulated conversion factor of $2.03 \mu \mathrm{m} /(\mathrm{mN} / \mathrm{m})$ it can be concluded that the simulations predicted the device behavior relatively well. 
(a)

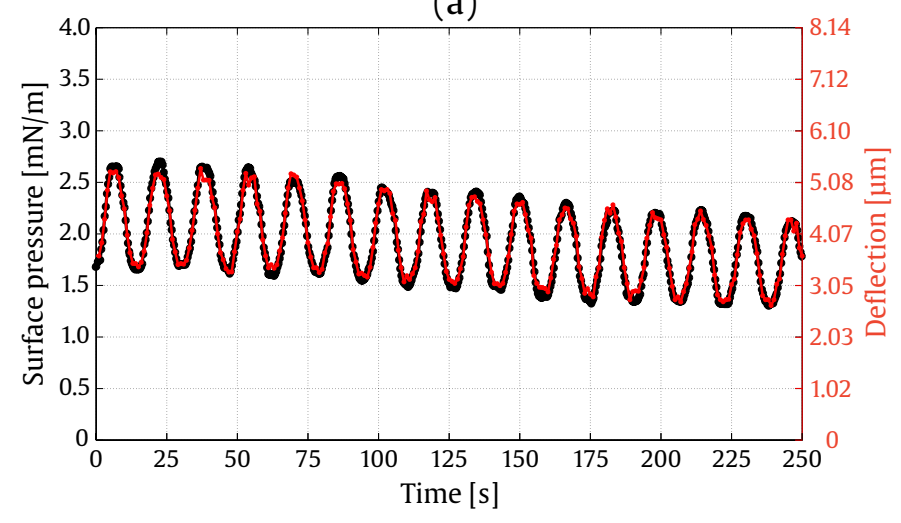

(b)
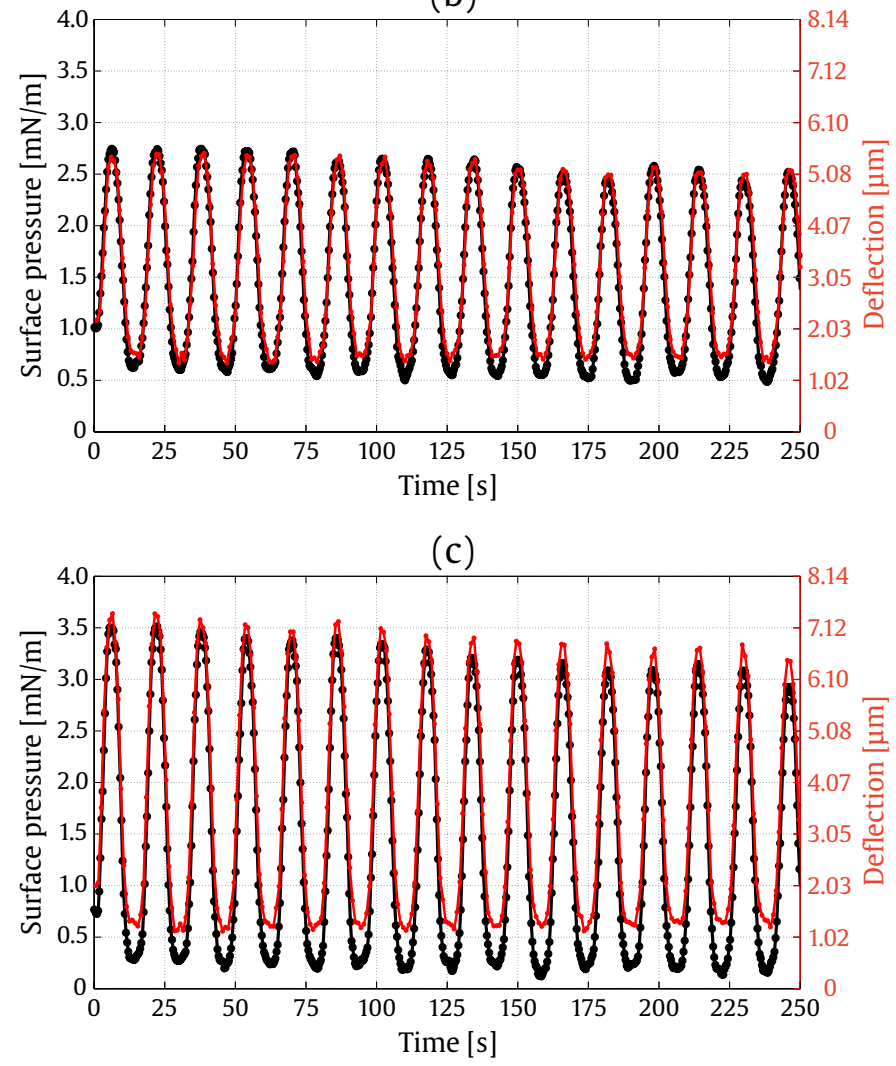

Figure 8: Measurement results showing the surface pressure measured by a Wilhelmy plate (black) and the tensiometer deflection (red) during three consecutive rapid local oscillation experiments. (a) 16 oscillations at $62.5 \mathrm{mHz}$ with $1 \%$ area change. (b) 16 oscillations at $62.5 \mathrm{mHz}$ with $2 \%$ area change. (c) 16 oscillations at $62.5 \mathrm{mHz}$ with $3 \%$ area change. 